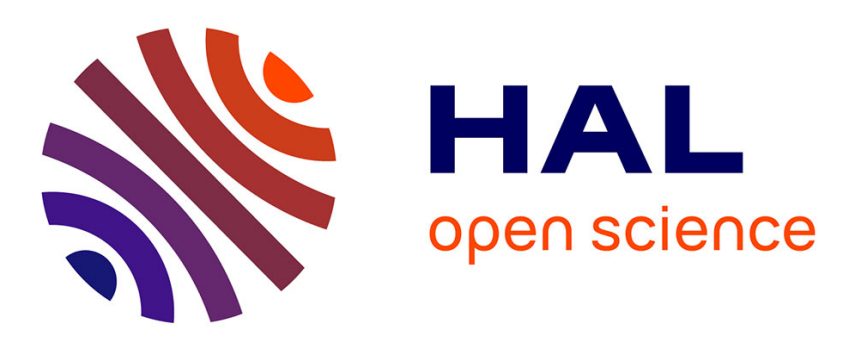

\title{
Tuning the nanostructure of highly functionalized silica using amphiphilic organosilanes: Curvature agent effects
}

Romain Besnard, Guilhem Arrachart, Julien Cambedouzou, S Pellet-Rostaing

\section{To cite this version:}

Romain Besnard, Guilhem Arrachart, Julien Cambedouzou, S Pellet-Rostaing. Tuning the nanostructure of highly functionalized silica using amphiphilic organosilanes: Curvature agent effects. Langmuir, 2016, 32 (18), pp.4624-4634. 10.1021/acs.langmuir.6b00589 . hal-01998424

\section{HAL Id: hal-01998424 https://hal.science/hal-01998424}

Submitted on 28 Oct 2021

HAL is a multi-disciplinary open access archive for the deposit and dissemination of scientific research documents, whether they are published or not. The documents may come from teaching and research institutions in France or abroad, or from public or private research centers.
L'archive ouverte pluridisciplinaire HAL, est destinée au dépôt et à la diffusion de documents scientifiques de niveau recherche, publiés ou non, émanant des établissements d'enseignement et de recherche français ou étrangers, des laboratoires publics ou privés. 


\section{Tuning the nanostructure of highly functionalized}

\section{silica using amphiphilic organosilanes: Curvature}

\section{agent effects.}

Romain Besnard, Guilhem Arrachart*, Julien Cambedouzou, ${ }^{*}$ Stéphane Pellet-Rostaing

Institut de Chimie Séparative de Marcoule, UMR 5257 CEA / CNRS / UM / ENSCM, BP17171, F-30207 Bagnols-sur-Cèze, France.

E-mail : guilhem.arrachart@cea.fr julien.cambedouzou@enscm.fr

Cerasomes / nanofibers / bilayers / blackberry-like particles / highly functionalized silica.

The self-assembly of amino-undecyl-triethoxysilane (AUT) as micelles in water is considered. The behavior of acid/AUT systems is governed by a complete proton transfer from the acid to the amine, leading to the formation of an ammonium head group. This moiety is responsible of the bending of the interface between the organic core of the micelles and the surrounding water. By playing with the size of the acid used as curvature agent, the amphiphilic behavior of the organosilane molecule may be adjusted. We follow the aggregation as the curvature agent size increases. This approach constitutes an efficient and original method in order to tune the nanostructure of highly functionalized silica at the early stage of the elaboration. Small angle X- 
ray scattering, wet scanning transmission electron microscopy, dynamic light scattering and complementary characterization techniques indicate that hybrid organic-inorganic planar objects and vesicles are obtained for smaller curvature agents. Increasing the size of the curvature agent results in a transition of the aggregation geometry from vesicles to cylindrical direct micelles, finally leading to nanofibers organized in a 2D hexagonal network resembling a "reverse MCM41 structure". A geometrical molecular self-assembly model is finally proposed, considering the dimensions of the surfactant tail and those of the head groups.

\section{Introduction}

The combination of inorganic and organic parts in hybrid materials allows associating an important variety of innovative properties, leading to a large application field. ${ }^{1-10}$ Among hybrid materials, ones of the most studied are organically functionalized silicas obtained by sol-gel process. From the surfactant-mediated route, different pathways exist in order to functionalize the matrix, involving the post-grafting of a functional alkoxysilane or the co-condensation during the silica preparation. Nevertheless, the literature displays important limitations like the weak functionalization ratio and the non-homogeneous grafting due to blocking effects at the entrance

of the pores. ${ }^{11}$ Such blocking effects also result in a limited accessibility to the functionalities, and represent a problem for some applications such as solid/liquid extraction, liquid waste processing, metal immobilization or catalysis.

Beyond these approaches, where external templating macromolecules or supramolecular assemblies were employed as the main structuring agent, it has been shown that nanostructured materials could also be obtained thanks to the auto-association properties of the organic units of 
organosilanes. ${ }^{12-14}$ Weak interactions such as hydrogen-bonding interactions of organic units are able to drive the aggregation of organosilane molecules and finally nano-structured materials can be elaborated without supplemental surfactant. ${ }^{15}$ An alternative procedure for elaborating functional silica materials presenting a high density of organic functions has been already described in a few studies, and consists in the use of amphiphilic organosilane molecules. ${ }^{16-19}$ Such molecules combine both condensable and functional sites around a long hydrophobic alkyl chain. Reverse cylindrical micelles or bilayers, respectively leading to $2 \mathrm{D}$ hexagonal or lamellar structures have been prepared with various amphiphilic organosilane molecules. ${ }^{20,21}$

The tuning of the meso-structure of hybrid silicas however remains a challenge in nanoscience and nanotechnology. Besides, the availability and the versatility of the functional organic moieties are also preponderant parameters for the performance of the material. The primary amino group is one of the most attractive surface functionalities owing to its use as chelating site for various metal cations, ${ }^{22,23}$ but also regarding its high versatility. Indeed, amino groups are useful for the introduction of various functionalities by chemical modification. ${ }^{22,24-26}$

The present work aims to establish a critical examination of the self-assembly of amino-undecyltriethoxysilane (AUT) in the presence of different agents able to catalyze the sol-gel reaction and also to form an ammonium polar head. The ability of this polar head to bend the interface between the aliphatic chains of the AUT and the surrounding water varies depending on the size of the associated agent. We therefore show that it is possible to tune the organization into bilayers, multibilayers vesicles, cylindrical direct micelles, or nanofibers by increasing the curvature agent size. Dynamic Light Scattering (DLS) and Wet Scanning Transmission Electron Microscopy (WetSTEM) highlight the shape and size of the particles contained in the precursor suspensions. The structural evolution according to the concentration of the AUT as well as the associated agent was 
followed by Small Angle X-ray Scattering (SAXS) experiments, and shows a swelling mechanism of the lamellar structure leading to multi-bilayer vesicles. We highlight by Transmission and Scanning Electron Microscopy (TEM or SEM) the effect of the curvature agent on the morphology of the final material. In particular, blackberry-like particles and organized nanofibers can show up depending on the composition. Finally, we propose a model describing the amphiphilic behavior of AUT molecules with regards to their associated curvature agents. This model turns out to be a relevant tool in order to predict the geometry of the aggregates depending on the size of the acid used as counter-ion.

\section{Experimental section}

\section{Technical details}

SAXS experiments were performed on a set-up operating in transmission geometry. A Mo anode associated to a Fox2D multi-shell mirror (XENOCS) delivers a collimated beam of wavelength $0.710 \AA$. Two sets of scatterless slits ${ }^{27}$ delimitate the beam to a square section of side length 0.8 mm. A MAR345 imaging plate detector allows simultaneously recording scattering vectors $\mathrm{q}$ ranging from $0.25 \mathrm{~nm}^{-1}$ up to $25 \mathrm{~nm}^{-1}$. Samples were placed in glass capillaries of $2 \mathrm{~mm}$ diameter. Absolute intensities were obtained by measuring a calibration sample of high density polyethylene (Goodfellow) for which the absolute scattering was already determined. All SAXS profiles are plotted in log-log scale in order to highlight the dependency of the intensity versus a power law of the scattering vector $\mathrm{q}$.

HRTEM was carried out at $200 \mathrm{kV}$ on a JEOL $2200 \mathrm{FS}$ microscope. Samples obtained after heat treatment were ground into a powder and dispersed in ethanol. Samples were then deposited on 
400-mesh carbon-coated copper grids. After ethanol evaporation, the grid was submitted to analysis.

SEM was performed on a FEI QUANTA 200 ESEM FEG operating at $15 \mathrm{kV}$ equipped with a Everhart-Thornley detector.

A FEI Quanta 200 ESEM FEG microscope operated at $30 \mathrm{keV}$ was used for Wet-STEM experiments. A Peltier stage and an inverse BSE detector are located below the final lens in the microscope chamber. A droplet of the diluted sample solution was deposited on a TEM copper grid coated with a holey carbon film. The grid was then placed on the Peltier stage previously cooled at $\mathrm{T}=2{ }^{\circ} \mathrm{C}$. In order to obtain a pure water vapor atmosphere in the ESEM chamber, an optimized pump down sequence was used to adjust the water partial pressure at $706 \mathrm{~Pa}: 12$ successive purges between $600 \mathrm{~Pa}$ and $1200 \mathrm{~Pa}$ lead to $99.5 \%$ water vapor in the ESEM chamber. Afterwards, the pressure is adjusted around $700 \mathrm{~Pa}$ to evaporate a very small amount of water from the droplet to adjust the water film thickness and allow sample observation of the sample through a water film.

TGA analyses were performed on a Setaram Setsys Evolution 18 instrument under air flow (20 mL. $\left.\min ^{-1}\right)$ with a heating rate of $\left(5^{\circ} \mathrm{C} \cdot \mathrm{min}^{-1}\right)$. The coupled mass spectrometer was a Hiden analytical QGA instrument.

Dynamic Light Scattering (DLS) measurements were performed on an ALV-CGS3 with a red laser beam $(\lambda=638.2 \mathrm{~nm})$. The scattering angle was set to $173^{\circ}$. The temperature of the sample environment was controlled and stabilized at $20^{\circ} \mathrm{C}$. Sample quartz tubes (square glass cells) were cleaned-up with ethanol and then dried. The solutions were diluted to meet the requirements of turbidity and viscosity for DLS measurement. 
Raman spectra were recorded on a Horiba Jobin Yvon LabRAM Aramis confocal Raman microscope, using an excitation wavelength of $\lambda=532 \mathrm{~nm}$ with a laser spot size of $\sim 1 \mu \mathrm{m}$ with an objective of X50 long working distance. The incident laser power was kept lower than $0.8 \mathrm{~mW}$ in order to avoid any heating or structural sample damage.

Solid-state Fourier transform infra-red (FT-IR) spectra were obtained using an ATR crystal on a Perkin-Elmer 100 spectrometer having a $4 \mathrm{~cm}^{-1}$ resolution.

The solid state ${ }^{29} \mathrm{Si}$ cross-polarization magic angle spinning (CP-MAS) nuclear magnetic resonance (NMR) spectra were recorded on a Bruker 400 ultrashield VS spectrometer equipped with a $4 \mathrm{~mm}$ MAS probe. The MAS spinning rate was $12 \mathrm{kHz}$. The typical contact time was of 2-3 $\mathrm{ms}$, and the typical recycle delay was 5-30 s.

Transmission optical microscopy was performed on a Zeiss Axio Imager A1m equipped with cross polarizers with a Epi DF reflector and an achromatic-aplanatic universal condenser $0.9 \mathrm{H}$. The stretching experiment was conducted on a flatted sample of $1 \mathrm{~mm}$ of thickness. The material has been manually stretched between two tongs until rupture of the material.

\section{Synthesis of amino-undecyl-triethoxysilane}

The organic precursor was synthesized in three steps, following a protocol adapted from the synthesis described in the literature ${ }^{1,28}$ : Bromoundecene (Alpha Aesar) was first mixed with triethoxysilane and Karstedt catalyst both purchased from ABCR to obtain bromoundecyltriethoxysilane. After purification, the latter molecule was then condensed with sodium azide (Sigma Aldrich) into acetonitrile to form azidoundecyl-triethoxysilane. Finally, the reduction of the azido group with Palladium $(10 \% \mathrm{Pd} / \mathrm{C})$ from Sigma Aldrich into anhydrous ethanol under hydrogen atmosphere led to aminoundecyltriethoxysilane.

\section{Materials syntheses and solution preparation}


The aim of the present study is to bring out the parameters which govern the self-assembly of amphiphilic organosilane molecules such as AUT. In a precedent study, the amphiphilic properties of dodecylamine, which contains the same functional group and almost the same alkyl chain than AUT was used as a structural agent of silica. ${ }^{29,30}$ The amphiphilic behavior of alkylammonium salts has been extensively studied. As mentioned in the works of Broome et al. ${ }^{31}$ who investigated the dodecylammonium chloride-water binary phase diagram, different regions were discovered in the phase diagram, including an isotropic solution, and different crystalline phases. The results obtained by Karlsson et al. ${ }^{32}$ showed that the phase behavior of carboxylic acid/alkylamine mixtures can also lead to such micellar aggregations. Then, inspired by the aforementioned works, Hartmann et al. ${ }^{33}$ applied a similar approach with a polymerizable organic counterion such as acrylic acid in order to get structured materials.

Taking into consideration the structural results obtained for dodecylamine, ${ }^{31,32}$ we chose to associate different acids with AUT molecules in order to improve the hydrophilicity of the amino group and the ability of the molecules to self-organize. By modifying the nature of the acid, we expect to tune the size of the amino polar head group and therefore the type of aggregation. We decided in the present study to experiment the addition of five different acids, namely carbon dioxide which forms a carbamic acid with the amine and leads to an ammonium carbamate dimer $\left(-\mathrm{NHCO}_{2}{ }^{-} \cdot{ }^{+} \mathrm{H}_{3} \mathrm{~N}-\right)$, hydrochloric acid $(\mathrm{HCl})$ at two different concentrations, acetic acid $\left(\mathrm{CH}_{3} \mathrm{CO}_{2} \mathrm{H}\right)$, pivalic acid $\left(\left(\mathrm{CH}_{3}\right)_{3} \mathrm{CCO}_{2} \mathrm{H}\right)$ and valproic acid $\left(\left(\mathrm{CH}_{3} \mathrm{CH}_{2} \mathrm{CH}_{2}\right)_{2} \mathrm{CHCO}_{2} \mathrm{H}\right)$. Therefore, 6 mixtures were prepared and respectively labelled $\mathrm{H} 0-\mathrm{CO}_{2}, \mathrm{H} 0-\mathrm{Cl}_{\mathrm{a}}$ and $\mathrm{H} 0-\mathrm{Cl} \mathrm{b}_{\mathrm{b}}, \mathrm{H} 0-$ Ac, H0-Piv and H0-Val. These preparations were compared to a reference $\mathrm{H} 0$ mixture prepared without acid. The hydrolysis and the condensation reaction were then performed by the combined 
action of water and the respective acid, except for $\mathrm{H} 0-\mathrm{CO}_{2}$ where hydrochloric acid is added in order to ensure the sol-gel reactions.

The concentration of the AUT molecule in all the compositions was fixed to $0.1 \mathrm{~mol} \cdot \mathrm{L}^{-1}$ in water. In order to avoid a pre-hydrolysis and a pre-organization of the organosilane molecules, the procedure first consists in the introduction of the curvature agent in a flask with the AUT, except for the hydrochloric acid which is added in the same time than the water. Before the water addition, samples are mixed at least $30 \mathrm{~s}$ with a vortex mixer. Then, water is added, and the mixtures are placed in a thermostatic orbital shaker at $30^{\circ} \mathrm{C}$ and $400 \mathrm{rpm}$ for 3 days. Table S1 in the Supporting Information sums up the composition of the samples.

Preparation of $\mathrm{HO}, \mathrm{HO}-\mathrm{Cl}_{a}$ and $\mathrm{HO}-\mathrm{Cl}_{b}$ mixtures

$\mathrm{H} 0, \mathrm{H} 0-\mathrm{Cl}_{\mathrm{a}}$ and $\mathrm{H} 0-\mathrm{Cl}_{\mathrm{b}}$ mixtures were prepared in the following route: $0.167 \mathrm{~g}$ of AUT are placed in flask and $5 \mathrm{~mL}$ of water, hydrochloric aqueous solution at $\mathrm{pH}=1.5$ and hydrochloric aqueous solution at $\mathrm{pH}=0.86$ are respectively added for $\mathrm{H} 0, \mathrm{H} 0-\mathrm{Cl}_{\mathrm{b}}$ and $\mathrm{H} 0-\mathrm{Cl}_{\mathrm{a}}$. Owing to the basic properties of the amino part of the AUT molecule ( $\mathrm{pKa}=10.6$, predicted with $\mathrm{ACD} / \mathrm{Labs}$ (PhysChem Suite 2014)), the $\mathrm{H} 0$ and $\mathrm{H} 0-\mathrm{Cl}_{\mathrm{b}}$ solutions are basic $(\mathrm{pH}=10.95$ and 10.22 respectively). In contrast, the hydrochloric aqueous solution of $\mathrm{pH} 0.86$ is acid enough for making the final solution acid $(\mathrm{pH}=1.38)$. The indexes $\mathrm{a}$ and $\mathrm{b}$ in $\mathrm{H} 0-\mathrm{Cl}_{\mathrm{a}}$ and $\mathrm{H} 0-\mathrm{Cl}_{\mathrm{b}}$ respectively refer to the acid or basic $\mathrm{pH}$ of the solution.

\section{Preparation of $\mathrm{HO}-\mathrm{CO}_{2}$}

$\mathrm{H} 0-\mathrm{CO}_{2}$ was synthesized according to the following procedure: $0.167 \mathrm{~g}$ of AUT are placed in a flask under $\mathrm{CO}_{2}$ flow during $15 \mathrm{~min}$. The formation of a gel is observed immediately. Then, $5 \mathrm{~mL}$ of a hydrochloric aqueous solution at $\mathrm{pH}=1.5$ were added. 
Preparation of H0-Ac, H0-Piv and H0-Val

H0-Ac, H0-Piv and H0-Val samples were elaborated as follows: 0.167g of AUT are placed in a flask and one equivalent of acetic acid, pivalic acid or valproic acid was respectively added for H0-Ac, H0-Piv and H0-Val. In the three cases, the mixture remains oily and homogeneous. Finally, $5 \mathrm{~mL}$ of water were added. The $\mathrm{pH}$ values for the resulting solutions were measured to $8.56,8.52$ and 8.22 for $\mathrm{H} 0-\mathrm{Ac}, \mathrm{H} 0-\mathrm{Piv}$ and $\mathrm{H} 0$-Val solutions respectively.

\section{Results and discussion}

The self-assembly of the amphiphilic AUT is studied as a function of the added curvature agents in water. The samples $\mathrm{H} 0, \mathrm{H} 0-\mathrm{CO}_{2}$ and $\mathrm{H} 0-\mathrm{Clb}$ quickly lead to precipitates, providing powders after filtration, washing, and drying. The solution $\mathrm{H} 0$-Cla is a white opaque suspension, while the solutions H0-Ac and H0-Piv look like clear and stable solutions even after several months. The direct aggregation and soft solid formation observed for $\mathrm{H} 0$-Val could be caused by the immiscility of the valproate ammonium complexes in water after the sol-gel process. The observations made on the different samples are reported in Table S2 in the Supporting Information.

The solid samples and the suspensions were characterized by SAXS experiments in order to check the meso-structure of the materials and the presence of micelles in the suspensions.

Figure 1a shows the SAXS profile of the $\mathrm{H} 0, \mathrm{H} 0-\mathrm{Cl}_{\mathrm{b}}$ and $\mathrm{H} 0-\mathrm{CO}_{2}$ materials. Their SAXS pattern exhibit four structure peaks located at $1.8 \mathrm{~nm}^{-1}, 3.6 \mathrm{~nm}^{-1}, 5.4 \mathrm{~nm}^{-1}$ and $7.2 \mathrm{~nm}^{-1}$. Such series of structure peaks positions correspond to a lamellar structure. Focusing on the small angle part of the three diagrams, the intensity follows a $\mathrm{q}^{-4}$ power law. Such a power law is known as a Porod law and is typical of sharp interfaces between the aggregates and the surrounding medium for a 
typical length scale larger than $10 \mathrm{~nm}$. The largest feature observable at around $15 \mathrm{~nm}^{-1}$ (typical distance of $0.42 \mathrm{~nm}$ in the real space) can be related to the typical mean lateral distance between two amphiphilic organosilane precursors. ${ }^{20}$ As already mentioned in a previous study, this experiment shows that the addition of hydrochloric acid or carbon dioxide as curvature agents leads to the same lamellar nanostructure. ${ }^{21}$ Considering the distances associated to the Bragg peaks characteristic of the lamellar order, the hypothesis of AUT bilayers sandwiching a polar head layer (as previously described in reference 21 ) is highly probable.
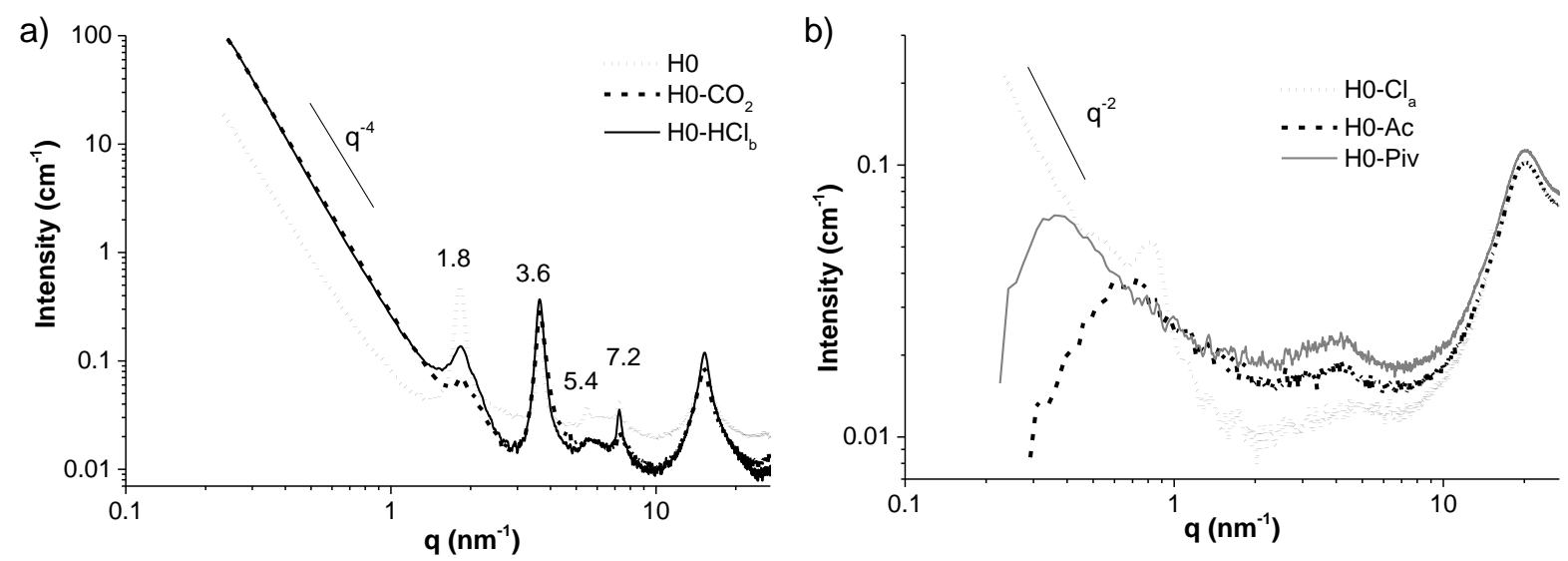

Figure 1. SAXS profiles of the a) $\mathrm{H} 0, \mathrm{H} 0-\mathrm{Cl}_{b}$ and $\mathrm{H} 0-\mathrm{CO}_{2}$ materials; b) $\mathrm{H} 0-\mathrm{Cl}_{\mathrm{a}}, \mathrm{H} 0-\mathrm{Ac}$ and $\mathrm{H} 0-$ Piv solutions.

Morphologically, the samples are very distinct (see Figure S1 in the Supporting Information). While the SEM images of $\mathrm{H} 0$ and $\mathrm{H} 0-\mathrm{CO}_{2}$ do not reveal any peculiar macro-structure (Figures S1a, S1b), those of $\mathrm{H} 0-\mathrm{Cl}_{\mathrm{b}}$ display a blackberry-like shape (Figure S1c). Such a blackberry morphology of organosilica hollow spheres had been already obtained by co-condensation of aminopropyltrietoxysilane with $\mathrm{TEOS}^{34}$ or from the condensation of TEOS around a cationic polymer. ${ }^{35}$ Nevertheless, it is the first observation to the best of our knowledge of such a morphology in a hybrid silica made without TEOS. The addition of hydrochloric acid could be a 
preponderant parameter for the morphology. The differences observed on the morphology of the $\mathrm{H} 0, \mathrm{H} 0-\mathrm{Cl}_{\mathrm{b}}$ and $\mathrm{H} 0-\mathrm{CO}_{2}$ materials are probably due to electrostatic interactions. Indeed, the interface between bilayers of the $\mathrm{H} 0-\mathrm{CO}_{2}$ materials is constituted principally of ammonium carbamate dimers $\left(\mathrm{R}-\mathrm{NHCO}_{2}-{ }^{-}{ }^{+} \mathrm{H}_{3} \mathrm{~N}-\mathrm{R}\right) \cdot{ }^{17}$

These dimers formed by attractive interactions are also present in the $\mathrm{H} 0$ material but in lower proportion. ${ }^{21}$ The partial addition of hydrochloric acid ( 0.3 eq./AUT) could perturb the formation of the ammonium carbamate dimers allowing the cohesion of the bilayers, and therefore generates a dendritic growth (inset in Figure S1c). The blackberry-like morphology of this sample could be related to this phenomenon.

The experiment involving a larger amount of hydrochloric acid in solution conducts to a white suspension called $\mathrm{H} 0-\mathrm{Cl}_{\mathrm{a}}$. The suspension is probably a direct consequence of the increase of electrostatic interactions which avoid the precipitation.

The same consequence is observed for the solution H0-Ac and H0-Piv, but these experiments lead to clear solutions. Figure $1 \mathrm{~b}$ shows the $\mathrm{SAXS}$ profiles of $\mathrm{H} 0-\mathrm{Cl}_{\mathrm{a}}, \mathrm{H} 0-\mathrm{Ac}$ and $\mathrm{H} 0-\mathrm{Piv}$ solutions. As can be seen by focusing on the small angle part of the three diagrams, the SAXS profile of the H0$\mathrm{Cl}_{\mathrm{a}}$ solution is different from those of $\mathrm{H} 0-\mathrm{Ac}$ and $\mathrm{H} 0-\mathrm{Piv}$. Indeed, while the $\mathrm{H} 0-\mathrm{Ac}$ and $\mathrm{H} 0-\mathrm{Piv}$ diagrams present a lump located respectively at around 0.6 and $0.4 \mathrm{~nm}^{-1}$, the $\mathrm{H} 0-\mathrm{Cl}_{\mathrm{a}} \mathrm{SAXS}$ profile exhibits an intensity following a $\mathrm{q}^{-2}$ power law and a structure peak at $0.8 \mathrm{~nm}^{-1}$. Such observations testify to the formation of planar objects stabilized in the solution and to typical interaction distances in the range of 7 to $8 \mathrm{~nm}$. Such interaction distances could be due to the typical distances between two bilayers separated by a water layer. According to this hypothesis, we assume the formation of muti-lamellar vesicles. 
Concerning H0-Ac and H0-Piv, the SAXS profiles shown in Figure $1 \mathrm{~b}$ do not provide an accurate insight into the aggregation state of these samples. The works of Karlsson et al. ${ }^{32}$ dealing with the amphiphilic behavior of dodecylamine which is very similar to the AUT molecule with acetic acid in equimolar ratio described the formation of direct cylindrical micelles. In order to get further insight into the structural organization and the micelle shape, an experiment aiming at swelling micelles with water was conducted. Mixtures with a growing amount of water $(50,60,70$ and 80 wt. \%) were performed leading to biphasic solutions. A phase separation is obtained with a minor white top phase and a major bottom clear phase. SAXS measurements are carried out on the major bottom clear phase.

First, the SAXS pattern of the sample containing $50 \mathrm{wt} . \%$ of water is composed of two broad structure peaks whose position correspond to a lamellar packing (Figure 2a). The large width of these peaks suggests a limited spatial correlation between the layers of the lamellar structure.

The SAXS profile of the sample containing $60 \%$ wt. of water presented in Figure $2 \mathrm{~b}$ features the superimposition of a lump at around 2-5 $\mathrm{nm}^{-1}$ and of two Bragg peaks corresponding to a lamellar structure whose lattice parameter is larger than expected for a molecular packing at the solid state $(3.9 \mathrm{~nm})$. Owing to the small width and the high intensity of these Bragg peaks, water insertion seems to result in a swelled lamellar structure defined on a large spatial area.

Then, by adding more water into the composition (Figures 2c), the SAXS profile exhibits a distorted structure in which the position of the first peak is shifted compared to the previous sample. Indeed, two broad peaks are clearly observable: the first one at $1 \mathrm{~nm}^{-1}$, and the second included between 2 and $5 \mathrm{~nm}^{-1}$. The increase of the water content also results in a change in the relative peaks' positions: the peak located at $1 \mathrm{~nm}^{-1}$ downshifts to $0.6 \mathrm{~nm}^{-1}$ and the broad one 
located at $2-5 \mathrm{~nm}^{-1}$ disappears (Figure $2 \mathrm{~d}$ and $2 \mathrm{e}$ ). Such observations feature a swelling of the initial bilayer organization as already depicted in the work of Bougis et al. ${ }^{36}$

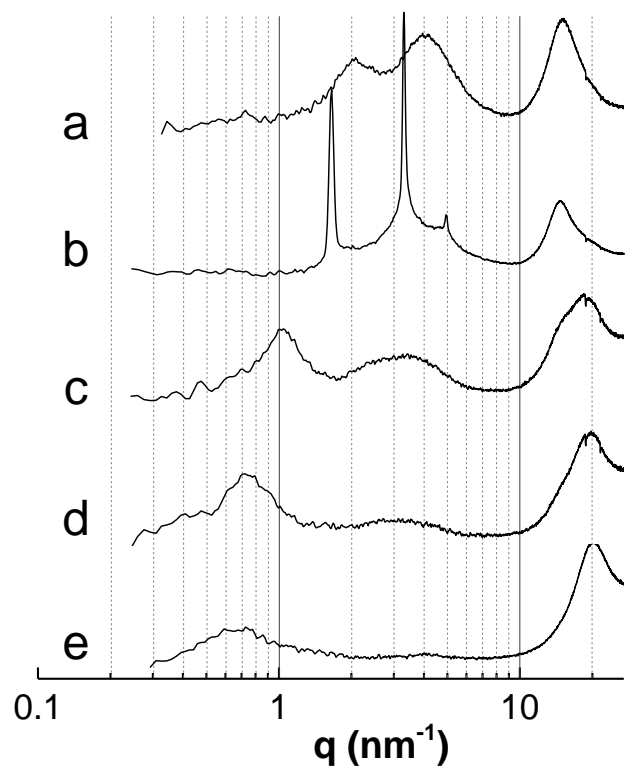

Figure 2. SAXS patterns of aqueous solutions composed of an equimolar ratio of AUT and acetic acid with different contents of water: a) 50 wt. \%, b) 60 wt. \%, c) 70 wt. \%, d) 80 wt. \%, e) 96.2 wt. \% (H0-Ac).

From this evolution of the SAXS profiles and considering the hydrophilicity of the ammonium polar head, a reasonable picture would be that water locates between bis-alkylammonium-silica bilayers. As the width of the water layer increases, the alkylammonium-silica bilayers are moving away from each other and do not interact anymore. Such multi-lamellar hybrid silica vesicles, also called cerasomes, have been already obtained by Katagiri et al. ${ }^{37}$ The description of these cerasomes establishes that the external layer is constituted of the silica part of the amphiphilic molecule. However, the results obtained from the present structural study and the high hydrophilicity of the ammonium acetate allow us to rule on a reverse cerasome structure, where 
the external layer is an ammonium species. A reasonable image of the reverse cerasome vesicles is schematized in Figure S2 in the Supporting Information.

The SAXS pattern of H0-Piv seems to exhibit the same profile than H0-Ac, except that the lump is located at $0.4 \mathrm{~nm}^{-1}$ instead of $0.6 \mathrm{~nm}^{-1}$, involving that the interlayer of water at the interface between the hybrid amino-silica bilayers would be larger.

The Wet-STEM images shown in Figure 3 are supplemental evidences of the vesicular aggregation. The $\mathrm{H} 0-\mathrm{Cl}_{\mathrm{a}}$ and $\mathrm{H} 0-\mathrm{Ac}$ solutions contain spherical objects of different sizes among which smaller ones are predominant (Figure $3 \mathrm{a}$ and $3 \mathrm{~b}$ ). In addition, the vesicles of diameter smaller than $100 \mathrm{~nm}$ contained in the H0-Ac solution seem denser than those of diameter $500 \mathrm{~nm}$ (Figure $3 b$ ). Therefore, the small particles are probably formed by more condensed vesicles than the larger ones. Comparatively, the H0-Piv solution seems to present homogeneous and monodisperse objects (Figure 3c). These objects have a typical diameter of $500 \mathrm{~nm}$ and seem to possess a low density, comparable to that of the larger vesicles in H0-Ac.
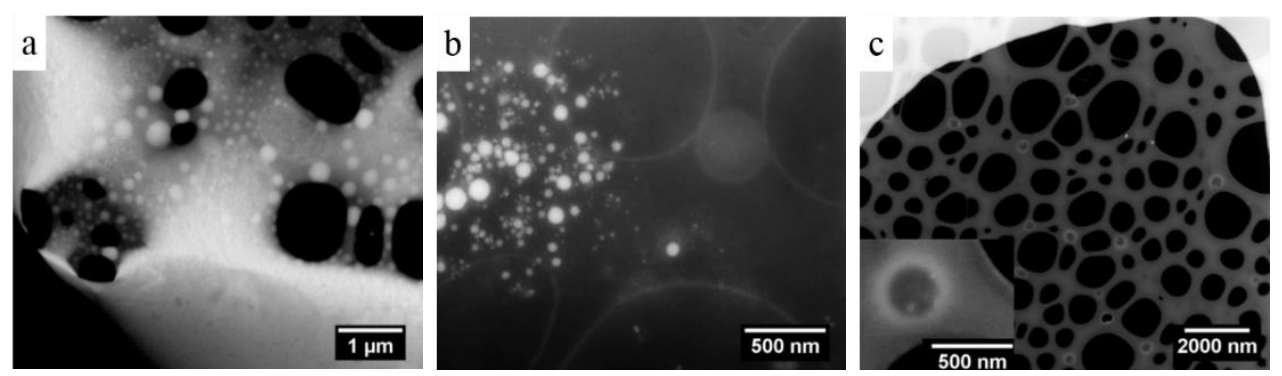

Figure 3. Wet-STEM images of suspensions a) $\mathrm{H} 0-\mathrm{Cl} a$ b) $\mathrm{H} 0-\mathrm{Ac}$, and c) H0-Piv.

The size distributions of the vesicles in the solutions were given by DLS. Table S3 in the Supporting Information shows the hydrodynamic radius, the area integration for each diameter and the polydispersity index (PDI) of $\mathrm{H} 0-\mathrm{Cl}_{\mathrm{a}}, \mathrm{H} 0-\mathrm{Ac}$ and $\mathrm{H} 0-\mathrm{Piv}$. The high PDI values involve that 
the object size distribution in solution is very large. The DLS theory assumes that aggregates are isotropic and monodisperse.

Therefore, discrepancies between the size distribution observed on Wet-STEM images and those obtained by DLS measurements are expected, and the resulting size values must therefore be handled carefully. In addition, the hydrodynamic diameter determined by DLS involves the hydration sphere around the micelles; the resulting hydrodynamic diameter is therefore necessarily larger than the diameter seen with microscopy techniques. However, the values are in the same range of size for both experiments. The results also show a significant ratio (close to 0.5 ) of objects whose size is included between 0.5 and $2 \mathrm{~nm}$ in the H0-Piv solution, suggesting that an important part of the scatterers is formed of oligomers or even monomers of AUT which would not be condensed with the rest of organosilanes. The DLS results on the H0-Ac solution also reveal such small aggregates but with a smaller proportion $(6 \%)$. The absence of a signal corresponding to the large particles $(500 \mathrm{~nm})$ seen in the H0-Ac solution during Wet-STEM measurements suggests that these objects are not much abundant compared to the smaller ones.

The large object size distribution in solution could be due to the simultaneous progress of the organization in micelles and that of sol-gel reactions. Indeed, the ethanol produced by hydrolysis and condensation reactions is progressively released in the medium. As the quantity of ethanol increases, the surface energy of the amphiphilic organosilane molecules decreases, favoring the formation of multi-bilayer vesicles.

To sum up this part, the incorporation of hydrochloric acid, acetic acid, and pivalic acid as curvature agents leads to vesicular aggregation. Therefore, the curvature agent size of the ammonium species is not sufficient to bend the interface enough to form cylindrical micelles. 
Aiming at confirming the trend of the self-assembly behavior, according to which the curvature of the aggregates increases with the size of the acid, an experiment was conducted with the valproic acid as curvature agent. The volume of this acid is indeed larger than that of the pivalic acid. The structural study of the soft H0-Val solid exhibits important differences compared to the previous samples. First, the slope at smaller angles on its SAXS pattern (Figure 4) does not present a $q^{-4}$ power law anymore, but follows a $\mathrm{q}^{-3}$ law. Such a power law is typical of an electron density gradient at the interface between the aggregates and the surrounding medium. It means that the interface is not sharp. Focusing at scattering vectors included between 1 and $10 \mathrm{~nm}^{-1}$ we note the presence of three well defined structure peaks at $2.4 \mathrm{~nm}^{-1}, 4.2 \mathrm{~nm}^{-1}$ and $4.8 \mathrm{~nm}^{-1}$. Such series of structure peaks could be the Bragg reflections on the planes (10), (11) and (20) of a 2D hexagonal structure constituted of organized cylinders of diameter $3 \mathrm{~nm}$. Cylinders of such a diameter could be formed by core-shell nano-fibers with silica as the core and undecyl-ammonium valproate as the shell.

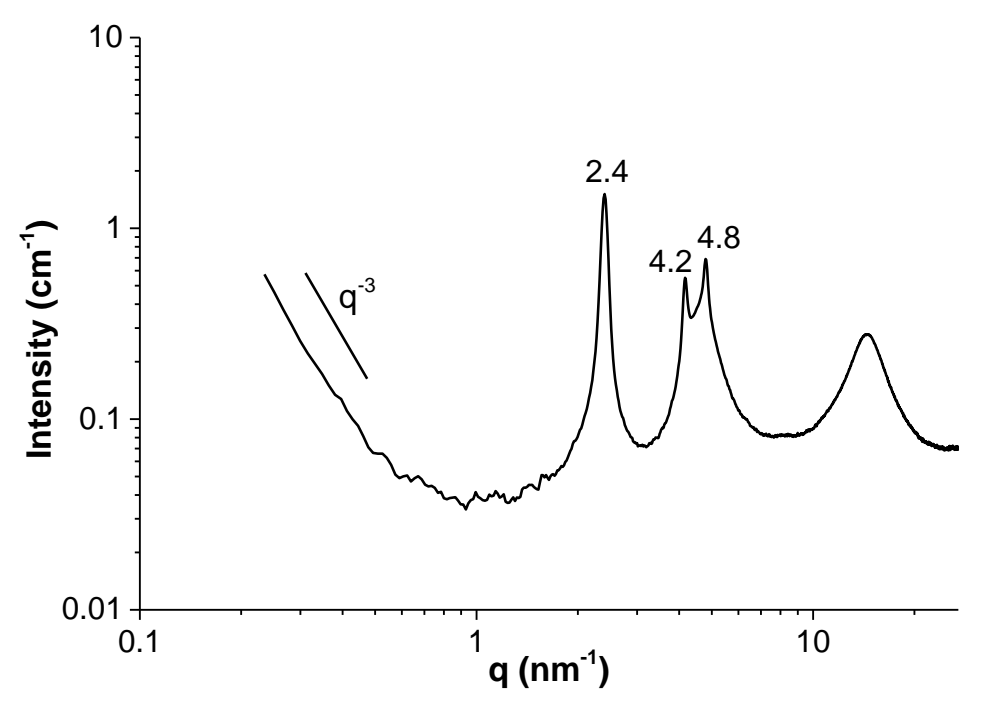

Figure 4. SAXS profile of the H0-Val material. 
The $\mathrm{q}^{-3}$ power law observed in the smallest angle region could be related to the blurry nature of the edges of the large objects made of aggregated cylinders.

At the meso and microscopic level, a clear demonstration of the anisotropic organization of the self-assembly of the AUT molecule with the valproic acid is the birefringence of the material observed by optical microscopy in cross-polarized light, as exemplified in Figure S3a in the Supporting Information. Under polarized light, optical microscopy observations show colored fringes in agreement with an optically anisotropic medium like a $2 \mathrm{D}$ hexagonal structure. Secondly, the material keeps its birefringence properties after stretching, meaning that the structure is not damaged (Figure S3b, S3c, S3d, and S3e see Supporting Information).

Further evidence of the auto-organization of the solid is the morphological aspect revealed by HRTEM microscopy observations. Figure 5 illustrates stripe patterns indicating that the hybrid nanofibers are stacked. The periodic distance of these stripe patterns is evaluated to $3 \mathrm{~nm}$ (Figure S4 see Supporting Information), in good agreement with the lattice parameter revealed by SAXS experiments.

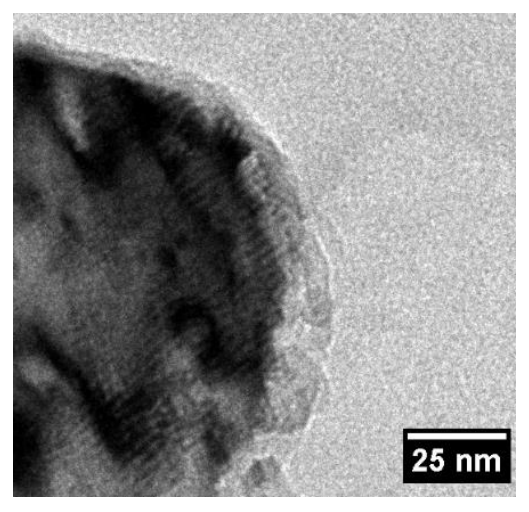

Figure 5. HRTEM image of the H0-Val material.

The soft aspect of the material could be the result of the weak cohesion between the molecules, which would not be well-condensed through Si-O-Si bridges. However, the ${ }^{29} \mathrm{Si}$ solid state NMR 
experiment allowed us to check the quantitative condensation ratio (Figure S5 in the Supporting Information). Indeed, the spectrum exhibits only two resonance signals. The first one, located at $\delta$ $=-60 \mathrm{ppm}$, is of small intensity and features the $\mathrm{T}^{2}$ units $\left(\mathrm{C}-\mathrm{SiOH}(\mathrm{OSi})_{2}\right)$ while the second one, at $\delta=-69 \mathrm{ppm}$, is much more intense, involving that the $\mathrm{T}^{3}$ units $\left(\mathrm{C}-\mathrm{Si}(\mathrm{OSi})_{3}\right)$ are preponderant. This result involves the presence of silsesquioxanes hybrid material. Otherwise, ${ }^{29} \mathrm{Si}$ solid state NMR clearly indicates that $\mathrm{Si}-\mathrm{C}$ bonds were retained within the sol-gel reaction, provided that only $\mathrm{T}^{\mathrm{x}}$ units were observed. Therefore, the condensation ratio is close to be complete, and the soft aspect of the material could be a direct consequence of its structural properties. The sliding of the unfixed fibers along themselves could provide such properties.

FTIR and Raman spectroscopies were conducted on the H0-Val material and the results were compared to those obtained with $\mathrm{H} 0$ in order to determine the presence of valproic acid in the $\mathrm{H} 0$ Val material (Figure 6).

1
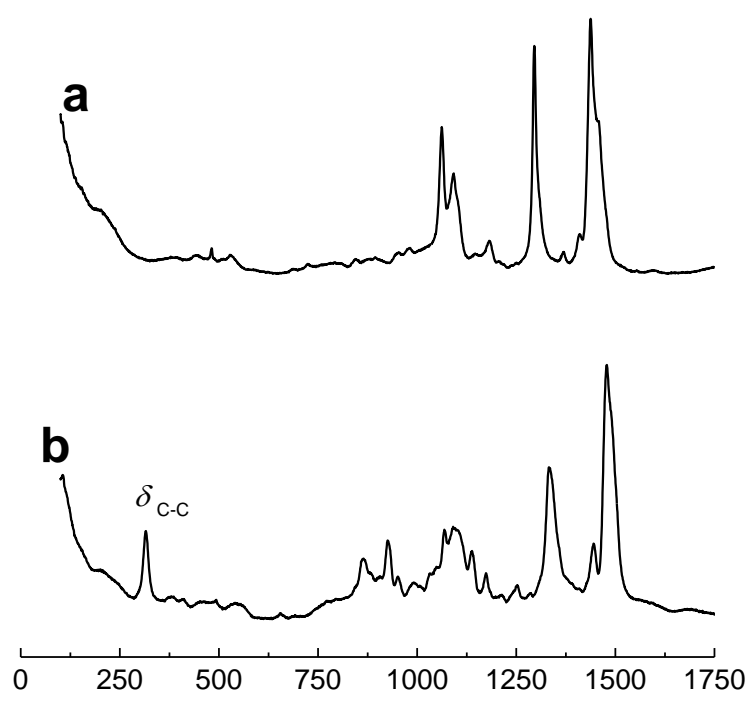

Raman shift $\left(\mathrm{cm}^{-1}\right)$
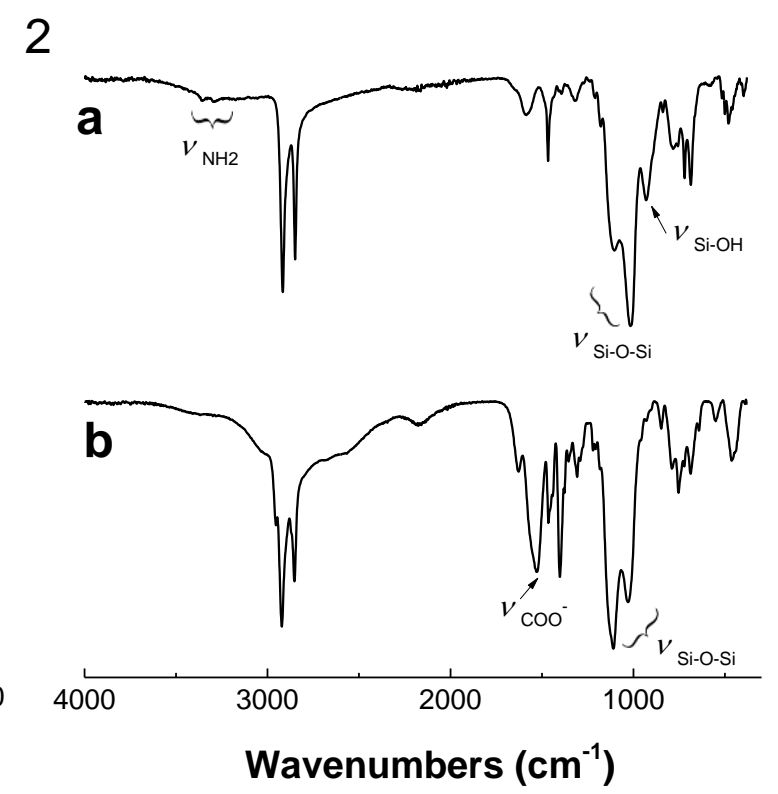

Figure 6. 1) Raman and 2) FTIR transmittance spectra of a) H0, and b) H0-Val material. 
These technical analyses also provide information in regards to the hydrolysis state of the siloxane part of the organosilane molecule in addition to the solid state NMR. By observing the Raman spectrum of the $\mathrm{H} 0$-Val material, we can note the presence of a band at $300 \mathrm{~cm}^{-1}$ that is missing in the Raman spectrum of H0 (Figure 6-1). This band features the bending vibration mode of the terminal propyl group of the valproic acid. Furthermore, the FTIR analysis presents important discrepancies between $\mathrm{H} 0$ and H0-Val materials (Figure 6-2). The vibration mode of the carboxyl group of carboxylic acid is usually expected close to $1700 \mathrm{~cm}^{-1}$, however no band is observed at this wavenumber. Nevertheless, the large band located at $1540 \mathrm{~cm}^{-1}$ testifies to the vibration mode of the carboxylate group. ${ }^{38}$ Concerning the $-\mathrm{NH}_{2}$ fragment in these materials, the FTIR analysis of the H0 material shows a weak double band located at around $3400-3250 \mathrm{~cm}^{-1}$, which can be attributed to the stretching vibration mode of $\mathrm{NH}_{2}$. This double band does not appear anymore on the FTIR pattern of $\mathrm{HO}$-Val, involving that the $\mathrm{NH}_{2}$ stretching mode vibration is not present due to the probable implication of this moiety in the formation of the ammonium species. Two other bands corresponding to the bending and the waging mode of $\mathrm{N}-\mathrm{H}$ bounds, respectively located at $1650 \mathrm{~cm}^{-1}$ and $730 \mathrm{~cm}^{-1}$, are visible on the $\mathrm{H} 0$ spectrum. Unfortunately, these bands are convoluted or hidden and cannot give supplemental insight about these fragments in the spectrum of H0-Val. The absence of the stretching mode of $\mathrm{NH}_{2}$ and the formation of carboxylate species may suggest the presence of ammonium carboxylate species in the H0-Val material.

The hydrolysis state of organosilane molecules can be checked by the disappearance of the band featuring the vibration mode of the ethoxy group of the organosilane molecule. Such a mode appears as a broad band located at $630 \mathrm{~cm}^{-1} \cdot{ }^{39}$ The Raman spectra of $\mathrm{H} 0$ and $\mathrm{H} 0$-Val do not exhibit such a band, meaning that the hydrolysis was well conducted in solution. Information about the condensation of the inorganic species can be also appreciated from the FTIR analysis following 
the $\mathrm{Si}-\mathrm{O}$ stretching modes $\mathrm{v}_{\mathrm{as}}(\mathrm{Si}-\mathrm{O})$ in the range from 1300 to $900 \mathrm{~cm}^{-1}$. Only $\mathrm{H} 0$ shows one band at $960 \mathrm{~cm}^{-1}$ corresponding to the silanol groups $v(\mathrm{SiOH})$, suggesting a better condensation ratio of the H0-Val material than for the H0 material. The most prominent Si-O-Si stretching bands are exhibited at 1025 and $1130 \mathrm{~cm}^{-1}$. The intensity ratio of these two bands, I $\left(1130 \mathrm{~cm}^{-1}\right) / \mathrm{I}\left(1025 \mathrm{~cm}^{-}\right.$ $\left.{ }^{1}\right)$ represents a measure of the state condensation of the material. ${ }^{40}$ The intensity of the $1120-1130$ $\mathrm{cm}^{-1}$ band is higher than that of the $1020-1040 \mathrm{~cm}^{-1}$ band for the H0-Val material, favoring the cube-like structure. ${ }^{41}$ The opposite trend is observed for the H0 material, indicating the predominance of ladder-like oligomers species. Other analyses have to be realized in order to confirm this hypothesis. Finally, the hydrolysis can be also confirmed on the SAXS profile by the absence of the $9 \mathrm{~nm}^{-1}$ shoulder, related to the effective hydrolysis of the silica matrix (Figure 4). ${ }^{20}$

In order to evaluate the ratio between organosilica units and valproic acid molecules, a thermogravimetric analysis was performed on the $\mathrm{H} 0$-Val material from $30{ }^{\circ} \mathrm{C}$ to $1000{ }^{\circ} \mathrm{C}$ under air flow. The resulting weight losses are shown in Figure S6 in the Supporting Information.

A weak water loss $(1.2 \mathrm{wt}$ \%) precedes the decomposition which occurs in two steps. The first one is principally linked to the decomposition of valproate species with a weight loss close to 26 wt. \%. The second one, evaluated at $56 \mathrm{wt} . \%$, is associated to the decomposition of the alkylamino part of the organosilica material. The residual mass $(16.8$ w. \%) after calcination can be considered as that of pure silica. Completely condensed silica presents a molar mass of $60 \mathrm{~g} \cdot \mathrm{mol}^{-1}$. Considering that each silicon atom of the silica originates from one precursor molecule of formula $\mathrm{O}_{1.5} \mathrm{Si}\left(\mathrm{CH}_{2}\right)_{11} \mathrm{NH}_{3}{ }^{+} \cdot \mathrm{xC}_{8} \mathrm{H}_{15} \mathrm{O}_{2}{ }^{-} \cdot \mathrm{yH}_{2} \mathrm{O}$, it can be deduced that the molar mass of this precursor compound is 357 g.mol ${ }^{-1}$. Moreover, the water loss (1.2 wt. \%) gives a water content of $4.3 \mathrm{~g}$ per mole of precursor compound. Considering that the species $\mathrm{O}_{1.5} \mathrm{Si}\left(\mathrm{CH}_{2}\right)_{11} \mathrm{NH}_{3}{ }^{+}$has a molar mass of 223 g.mol ${ }^{-1}$ and that the rest is constituted of pure valproate $\left(\mathrm{C}_{8} \mathrm{H}_{15} \mathrm{O}_{2}{ }^{-}\right)$of molar mass 143 g.mol ${ }^{-}$ 
${ }^{1}$, the formula of the precursor compound can be resolved as $\mathrm{O}_{1.5} \mathrm{Si}_{(}\left(\mathrm{CH}_{2}\right)_{11} \mathrm{NH}_{3}{ }^{+} \cdot 0.91 \mathrm{C}_{8} \mathrm{H}_{15} \mathrm{O}_{2}{ }^{-}$ $\cdot 0.09 \mathrm{HO}^{-} \cdot 0.15 \mathrm{H}_{2} \mathrm{O}$. The minimum ratio between the valproic acid and the organosilica unit is thus close to 1 .

Otherwise, such a water ratio involves that the hydration of the polar head is negligible in the residual solid and suggests that water is not a preponderant parameter in the self-assembly of the organosilane molecules in these conditions.

The results obtained from the structural and composition study allow us to give a picture of the nanofibers and their organization in the material, which is illustrated in Figure 7.

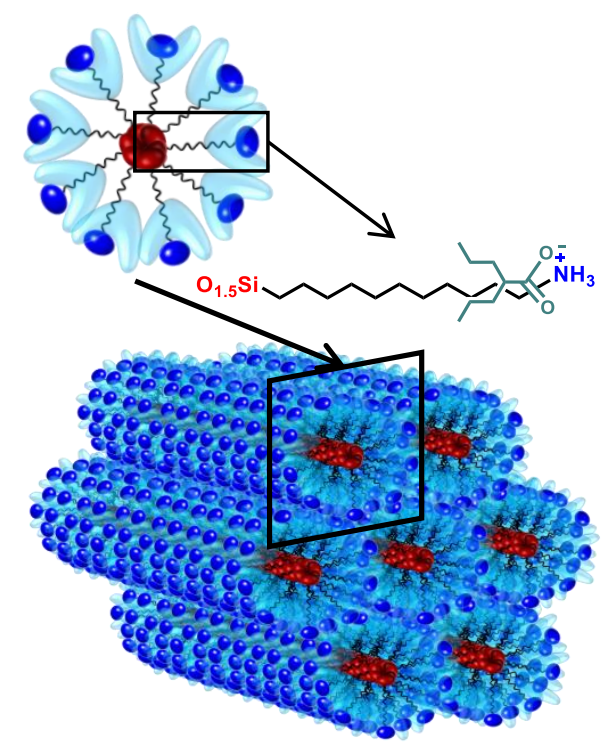

Figure 7. Schematic representation of organized core-shell silica nano-fibers formed by the selfassembly of the AUT in presence of the valproic acid as curvature agent.

\section{Self-assembly Mechanism}


Planar objects, vesicles and even rod-like structures have been prepared in accordance to the approach developed in this article.

Similarly, rod-like structures had been already observed by Kaneko et al. ${ }^{42}$ on a material prepared from aminopropyl-trimethoxysilane (APTMOS) and hydrochloric acid as sol-gel catalyst. Firstly, they described this network as a bilayer structure with an inclined parallel arrangement. They studied in depth the structure and concluded to a rod-like structure with a Si-O-Si framework at the core to form a hexagonal phase ${ }^{43}$ Finally, owing to the lattice parameter and the condensation ratio, they suggested a twisted ladder-like polymer with $\mathrm{O}_{x} \mathrm{Si}_{-}-\mathrm{NH}_{3}{ }^{+} \mathrm{Cl}^{-}$as monomer unit to form rigid rod-like structure in the solid state. The resulting arrangement of such aggregates is a hexagonally stacked structure. ${ }^{44}$ A study aimed at determining the dependence between structural aggregation and sol-gel catalyst revealed that the $\mathrm{pKa}$ of the acid is an important factor, leading to a rigid cage-like structure for higher $\mathrm{pKa}$ and to a rod-like structure for lower $\mathrm{pKa} .{ }^{44}$

In our study, the experiment involving the hydrochloric acid leads to a lamellar structure. The morphology and mesostructure evolution for $\mathrm{H} 0-\mathrm{Cl}_{\mathrm{a}}$ and $\mathrm{H} 0-\mathrm{Cl}_{\mathrm{b}}$ is linked to a catalytic process related to the acidic $(\mathrm{pH}=1.38)$ or basic $(\mathrm{pH}=10.22)$ starting solution involved in the sol-gel reaction. ${ }^{21}$ In addition, the pKa values of catalysts (acetic acid, pivalic acid and valproic acid) are very similar (from 4.6 to 5). However, in our case, we obtained different kinds of aggregation, involving that the self-assembly is not likely to be governed by the catalytic process.

The theoretical approach of the aggregation prediction requires a hydrophobic favorable contribution. The APTMOS alkyl chain studied by Kaneko et al. is composed of only 3 carbon atoms, therefore the amphiphilic properties of such a molecule are very weak, and the structure is influenced by the catalytic process. In our case, the long alkyl chain composed of 11 carbon atoms 
suggests a hydrophobic contribution. For that purpose, we propose a self-assembly mechanism based on the amphiphilic properties of the AUT molecules.

To predict the kind of aggregation (direct or reverse), the shape (sphere, cylinders, vesicles, etc...), and the size of aggregates, the scientific community specialized in colloids and emulsions conceptualized the aggregation by expressing the so-called "packing parameter". This term refers to the optimal aggregation geometry for a set of molecular parameters and intensive variables (such as temperature or ionic strength) through free energy minimization arguments. ${ }^{45}$ Three terms are required to express this packing parameter $P_{0}$ : the first is the volume $V_{c}$ of the hydrophobic portion of the surfactant, the second is the length of the hydrophobic chain $l_{c}$ and finally the third is the effective area $a_{0}$ per head group.

$$
P_{0}=\frac{V_{c}}{a_{0} l_{c}}
$$

The most problematic term in the definition of the packing parameter is the area per polar head $a_{0}$. For ionic surfactants, as in our case, $a_{0}$ depends on both electrolyte and surfactant concentration. The potential hydration of the polar head has to be also taken into account. It increases the polar head area according to the number of considered water molecules and therefore influences the apparent polar head area (a). For these reasons, it is difficult to make an exact calculation of the apparent packing parameter $P$ in a given system. However, we can predict the trends in $a$, and therefore $P$, for a specific change in the system using the expression:

$P=\frac{V_{c}}{a l_{c}}$

The siloxane part of the organosilane surfactant molecule is a supplemental problem in the prediction. Indeed, in the case of non-hydrolyzed siloxane part (tri-ethoxysilane), the polarity is weak and this segment should therefore be considered as hydrophobic in the calculation of the 
packing parameter. Otherwise, if the siloxane part is hydrolyzed (tri-hydroxylsilane) or condensed, it should be considered as hydrophilic in the calculation of the packing parameter. Therefore, the siloxane part of the organosilane surfactant can participate to the governing of the self-assembly as function of its hydrolysis state. In our study, we want to predict the final state of organization of the system. Therefore, we consider the siloxane part of the molecules as hydrolyzed or condensed. In this approach, the objective was to give the trend of the molecular self-assembly; owing to the weak volume and the invariability of the polar siloxane head group compared to the volume of the ammonium polar head group, we chose to ignore it in the calculation of the packing parameter. In other words, we consider only a simple undecylammonium molecule and its counter ion in the calculation model.

In this study, we experiment the addition of five different acids in identical aqueous solutions containing the amphiphilic organosilane surfactant. Although the medium is perpetually modified during the progress of the sol-gel reactions, we consider that the medium evolves similarly for each system. Therein, the assumption that the volume and the length of the hydrophobic chain remain constant in all systems can be made.

In order to estimate the packing parameter values, we calculated the molecular parameters with an equimolar ratio of curvature agent and amphiphilic molecule. The length of a fully extended alkyl chain of 11 carbon atoms without terminal methyl group is estimated at $1.397 \mathrm{~nm}$ according to Tanford. Its corresponding volume is $0.297 \mathrm{~nm}^{3}$. The ammonium polar heads have been considered as simple spheres, and their volume $\left(V_{0}\right)$ has been calculated thanks to Fedors' tables ${ }^{46}$ by summing the fragments constituting the counter ion. From these volumes, the radius $\left(R_{0}\right)$, the area $\left(a_{0}\right)$ and the packing parameter were deduced. For information, the area of a condensed siloxane head group was calculated to $0.145 \mathrm{~nm}^{2}$, which is very close to that of the polar head 
corresponding to the amino group. Figure S7 in the Supporting Information represents different possible geometries of aggregation depending on the packing parameter calculated for the surfactant and its curvature agent. The calculated values are reported in Table S4 in the Supporting Information.

By summing the volume of surrounding water molecules and the polar head volume, we take into account the hydration of the polar head in the apparent area calculation $(a)$. We estimated the number of water molecules surrounding the polar head between 0 and 6 .

We represented the different values of the apparent packing parameter $P$ as a function of the polar head area $\left(a_{0}\right)$ for different quantities of surrounding water molecules in Figure 8.

The graphical prediction highlights the reduction of the range of packing parameter values as ammonium head area increases. This evolution comes from the comparable molecular area of water and amine, ammonium carbamate or ammonium chloride. As the curvature agent increases, the molecular area of water becomes negligible, reducing the effect on the packing parameter values. Let us first consider the system without curvature agent. We can note that the area of the siloxane part is close to that of the amino part. We therefore expect that the equilibration between the two heads leads to planar objects and to a packing parameter value close to 1 . However the calculated value without water molecule gives $P_{0}=1.76$ (see Table S4 in the Supporting Information). The approximation that we made by not taking into account the siloxane head group in front of the amine without curvature agent probably introduces a divergence in the calculation in the case of the system without curvature agent. As proposed by the predictive molecular approach, the self-assembly of AUT could lead to the formation of lamellae or vesicles for the carbon dioxide and the hydrochloric acid as curvature agents, which is in agreement with the experimental results. 


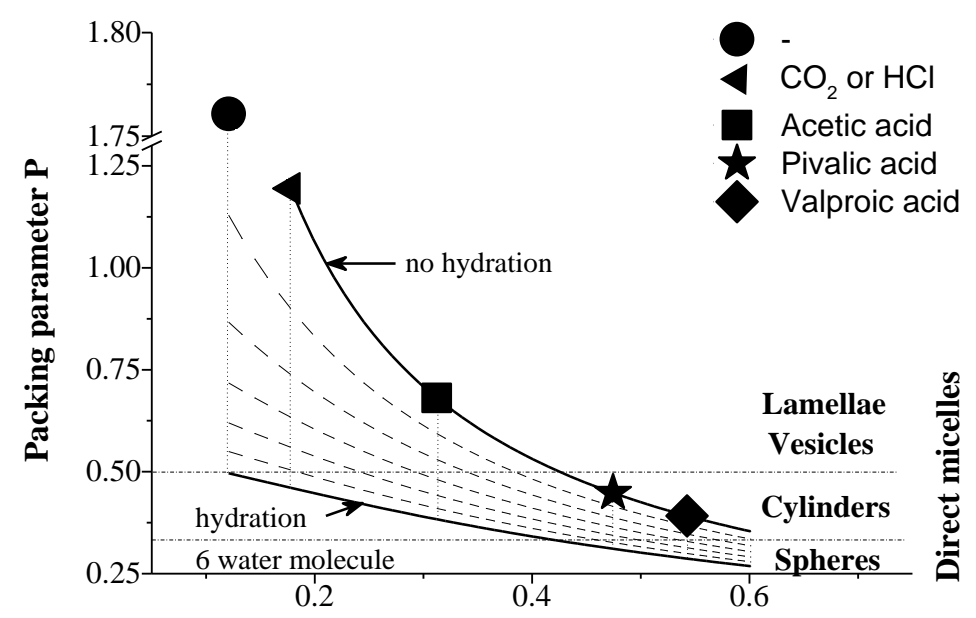

Ammonium head area $\left(\mathbf{n m}^{2}\right)$

Figure 8. Prediction of the packing parameter value $\mathrm{P}$ as function of the ammonium head area, with the polar head surrounded by 0 to 6 molecules of water. The different complexes acid/AUT were placed on the diagram in the hypothesis of dehydrated polar heads.

In addition, according to the counterion concentration, the structure evolves from a layered powder to a vesicular system, involving that the organosilane behaves as a surfactant in regard to the surrounding media.

Furthermore, either vesicles or direct cylindrical micelles are expected in mixtures involving acetic acid as curvature agent. Moreover, the ammonium acetate polar head is likely to be surrounded by a hydration sphere composed between three and four water molecules. ${ }^{32}$ The calculation gives for $\mathrm{H} 0$-Ac a polar head area value included between 0.44 and $0.48 \mathrm{~nm}^{2}$, which is close to the expected value described in the works of Karlsson et al. ${ }^{32}$ Such a value gives a calculated packing parameter close to 0.5 , which corresponds to the limit between planar aggregates and cylindrical micelles. A vesicular aggregation being observed experimentally, our model is coherent with experimental observations, albeit at the limit. 
The calculated packing parameter value of AUT with the pivalic acid as curvature agent predicts the formation of cylindrical direct micelles $(\mathrm{P}=0.37)$. However, the experiments show vesicles, suggesting a higher value typically beyond 0.5 . Moreover, the vesicular aggregation involves also that the ammonium pivalate polar head is surrounded by a hydration sphere, which tends to intensify the deviation compared to the calculation.

Concerning the sample involving valproic acid as curvature agent, we demonstrated by thermal analysis that the ammonium valproate polar head is hydrated by less than one water molecule. In this case we calculate a packing parameter value of 0.39 , corresponding to the formation of cylindrical direct micelles in good agreement with our experimental results.

Consequently, the packing parameter we calculated allowed us to predict the different geometries of aggregation determined experimentally, except concerning the pivalic acid, for which the calculated packing parameter (0.37) slightly differs from the expected one $(>0.5)$. Considering the simple hypotheses we made and the level of uncertainty on the molecular configuration when calculating the packing parameters, the model proposed here turns out to be an interesting tool in order to explain and anticipate the aggregation geometry of bola-amphiphilic molecules as the ones considered in this study. Nevertheless, this prediction model remains perfectible. The improvement should include a term corresponding to the contribution of the siloxane part of the molecule. It could also be refined by considering the dipole moment of the different salts for the calculation of the polar head area. Usually, the theoretical approach of the aggregation prediction of bola-amphiphilic molecules considered two associative polar head groups surrounding the hydrophobic chain. ${ }^{47}$ Here, they are dissociated to form the silica matrix and the ammonium salt. Therefore, the improvement of the prediction model has to consider the polar head groups as competitive in the prediction model. 
Otherwise, while the polar head area of ammonium pivalate is close to that of ammonium valproate (Table S4 in the Supporting Information), the amphiphilic behavior study in water did not allow getting intermediate aggregates able to discriminate the critical area head group between vesicles and cylinders. The vesicular organizations observed for $\mathrm{H} 0$-Ac and H0-Piv involve electrostatic interactions between the ammonium species that water stabilizes. In the case of $\mathrm{H} 0$-Val, the content of water in the aggregates is negligible. Thereby, we cannot strictly compare their aggregation. For that purpose, we experiment the effect of organic solvent as medium in order to free the aggregation of the water effect.

\section{Solvent effect.}

Our study focused on systems aggregating in aqueous media. Water is of course a fundamental constituent of the mixtures regarding the aggregation geometry. In the following, we studied the structure of samples in which water was replaced by an organic solvent. These experiments are aimed at determining the role played by water in the organization process. Di-isopropylether was selected for its low polarity and its aproticity. We compared the structures obtained considering different acids, namely acetic acid, pivalic acid and valproic acid (Figure 9). The self-assembly with the acetic acid as curvature agent leads to white powders. The resulting material from the self-assembly with the pivalic or the valproic acid are clear soft solids. As revealed by SAXS experiment in Figure 9, the organization changes depending on the carboxylic acid used as curvature agent. While the experiment in water led to a vesicular system for the sample made with acetic acid, the sample made in di-isopropylether led to a lamellar structure as can be seen in Figure 9a). This result demonstrates that the solvent modifies the interactions between the polar heads at the interfaces. 


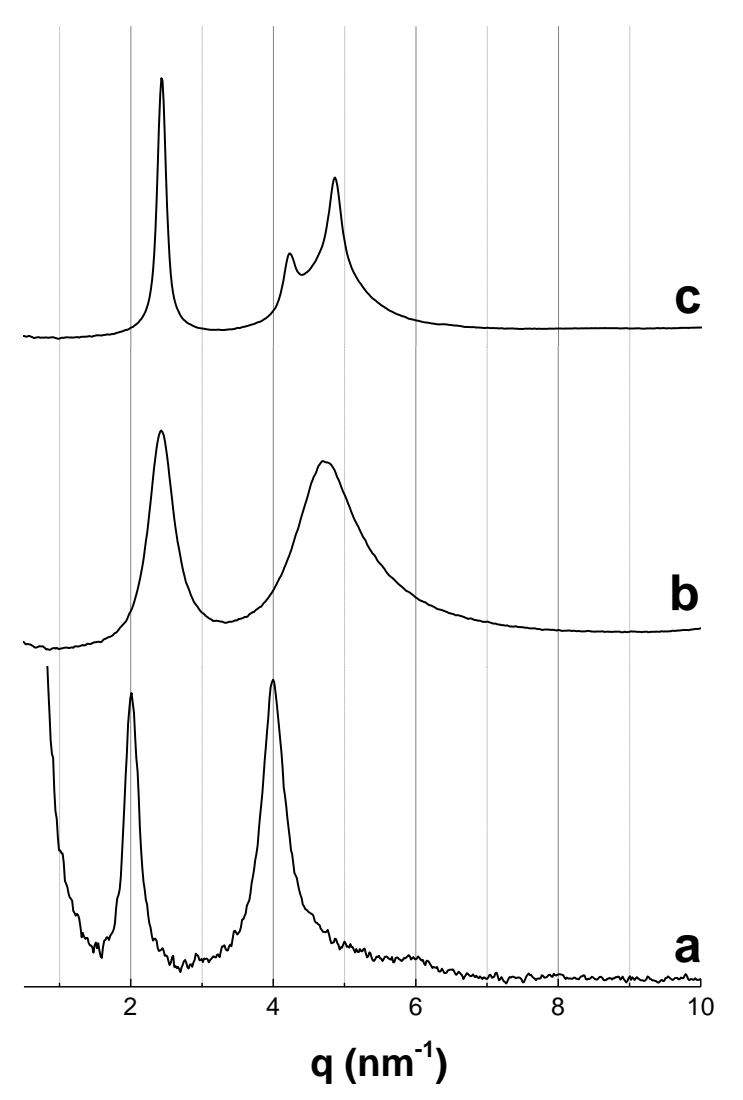

Figure 9. Linear-linear SAXS profiles of materials prepared with a) the acetic acid, b) the pivalic acid and c) the valproic acid as curvature agent in di-isopropylether.

In the case of water, the hydration of the polar ammonium acetate group could allow swelling the lamellar structure adopting a multi-bilayer vesicles organization. In the experience involving diisopropylether, the lattice parameter determined from the first structure peak located at $2.00 \mathrm{~nm}^{-1}$ is slightly smaller to that found for the $\mathrm{H} 0$ material, which could be due to the absence of water. The use of the valproic acid led to the same SAXS profile (Figure 9c) than that of H0-Val prepared in water. As expected, the same 2D hexagonal structure is obtained with a lattice parameter estimated at $3 \mathrm{~nm}$. This result confirms that water does not govern the assembly of such alkylammonium valproate species. 
The material prepared with the pivalic acid presents a SAXS profile exhibiting a first broad structure peak at $\mathrm{q}_{1}=2.43 \mathrm{~nm}^{-1}$ and a second at $\mathrm{q}_{2}=4.74 \mathrm{~nm}^{-1}$, which is twice larger (Figure $9 \mathrm{~b}$ ). The ratio $\mathrm{q}_{2} / \mathrm{q}_{1}=1.95$ fits with a ratio close to that expected for a lamellar phase $(\mathrm{q}(002) / \mathrm{q}(001)$ $=2$ ). However, the position of the first peak is close to that of the material involving the valproic acid, which is made of 2D hexagonal arrays of cylinders. In addition, owing to the broadening of the first peak, we assume that the second peak could be a convolution of several broad peaks. Pivalic acid as curvature agent could therefore lead to an intermediate aggregation state between planar objects and cylindrical direct micelles. A reasonable image of such aggregates could be ribbons, as depicted in Figure 10. Such an organization is close to that given by the prediction model, which gives a packing parameter value close to an intermediate state between vesicles and cylinders.

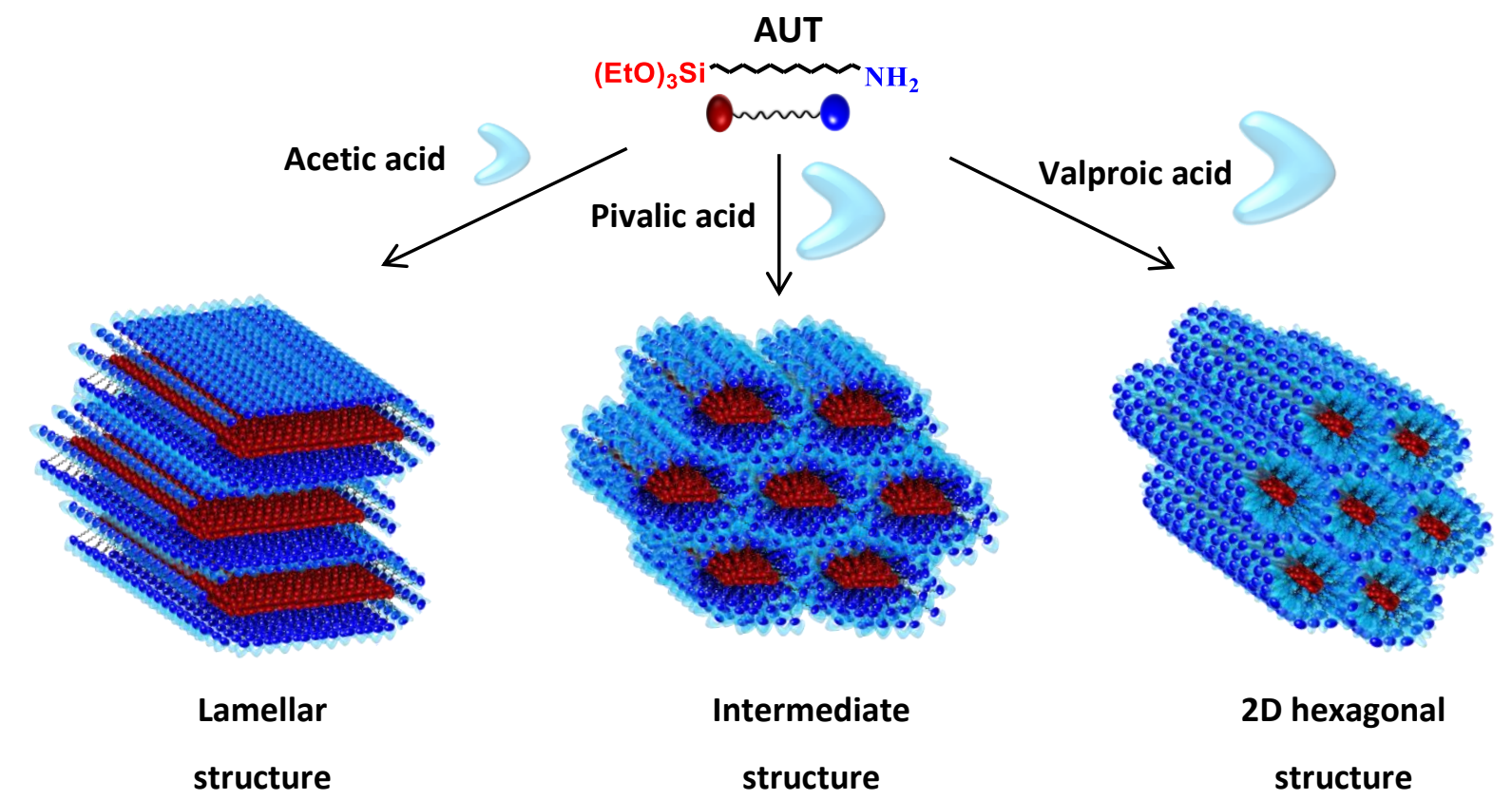

Figure 10. Schematic representation of the structural evolution depending of the curvature agent in water free media. 
As for material made with the acetic acid, the self-assembly with the pivalic acid is therefore affected by the solvation of the polar head. Indeed, while multi-bilayer vesicles are obtained in water, the use of another solvent results in another aggregation geometry.

\section{Conclusion}

By using a simple amphiphilic organosilane and a counterion as curvature agent, we show in this article that the shape of the aggregates can be tuned as well as the nanostructure of the highly functionalized silicas finally obtained. We highlight in this article the mechanisms governing the organization of the amphiphilic organosilane molecules. As classical amphiphilic molecules, their organosilane counterpart are affected by the kind of counter ion and more specifically their size. This study also shows the role of the interactions at the interface between the bilayers, leading to a swelling in the case of repulsive interactions or a collapsing in the opposite case. We propose a self-assembly theoretical approach considering the contributions arising from the surfactant tail and the head groups, which turns out to represent a relevant tool in order to understand the selfassembly mechanism as function of the curvature agents. In the second part of this study we explore the preponderant effects on the morphology due to the change of solvent. 


\section{Associated content}

\section{Supporting Information}

Tables S1 - S4 and Figures S1 - S7. This material is available free of charge via the Internet at http://pubs.acs.org.

\section{Author information}

\section{Corresponding Author}

*E-mail: guilhem.arrachart@cea.fr ; julien.cambedouzou@enscm.fr.

\section{Notes}

The authors declare no competing financial interest.

\section{Acknowledgments}

The authors acknowledge J. Ravaux for assistance on Wet-STEM experiments, X. Le Goff for TEM experiments, A. Jonchère for support in spectroscopic measurements, and C. Rey for TGA experiments. 


\section{References}

(1) Lambert, S.; Tran, K. Y.; Arrachart, G.; Noville, F.; Henrist, C.; Bied, C.; Moreau, J. J. E.; Wong Chi Man, M.; Heinrichs, B. Tailor-Made Morphologies for Pd/SiO2 Catalysts through SolGel Process with Various Silylated Ligands. Microporous Mesoporous Mater. 2008, 115 (3), 609617.

(2) Trong On, D.; Desplantier-Giscard, D.; Danumah, C.; Kaliaguine, S. Perspectives in Catalytic Applications of Mesostructured Materials. Appl. Catal. Gen. 2001, 222 (1-2), 299-357.

(3) Otani, W.; Kinbara, K.; Zhang, Q.; Ariga, K.; Aida, T. Catalysis of a Peptidic Micellar Assembly Covalently Immobilized within Mesoporous Silica Channels: Importance of Amphiphilic Spatial Design. Chem Eur J 2007, 13 (6), 1731-1736.

(4) Bourg, S.; Broudic, J.-C.; Conocar, O.; Moreau, J. J. E.; Meyer, D.; Wong Chi Man, M. Tailoring of Organically Modified Silicas for the Solid-Liquid Extraction of Actinides. Chem. Mater. 2001, 13 (2), 491-499.

(5) Arrachart, G.; Karatchevtseva, I.; Cassidy, D. J.; Triani, G.; Bartlett, J. R.; Chi Man, M.

W. Synthesis and Characterisation of Carboxylate-Terminated Silica Nanohybrid Powders and Thin Films. J Mater Chem 2008, 18 (31), 3643.

(6) Delchet, C.; Tokarev, A.; Dumail, X.; Toquer, G.; Barré, Y.; Guari, Y.; Guerin, C.; Larionova, J.; Grandjean, A. Extraction of Radioactive Cesium Using Innovative Functionalized Porous Materials. RSC Adv 2012, 5707-5716. 
(7) Oviatt, H.; Shea, K.; Kalluri, S.; Shi, Y.; Steier, W.; Dalton, L. Applications of Organic Bridged Polysilsesquioxane Xerogels to Nonlinear-Optical Materials by the Sol-Gel Method. Chem Mat 1995, 7 (3), 493-498.

(8) Hsiue, G. H.; Lee, R. H.; Jeng, R. J. A New Class of Organic-Inorganic Sol-Gel Materials for Second-Order Nonlinear Optics. Chem Mat 1997, 9 (4), 883-888.

(9) Lebeau, B.; Brasselet, S.; Zyss, J.; Sanchez, C. Design, Characterization, and Processing of Hybrid Organic-Inorganic Coatings with Very High Second-Order Optical Nonlinearities. Chem Mat 1997, 9 (4), 1012-1020.

(10) Chen, Z.; Samuelson, L.; Akkara, J.; Kaplan, D.; Gao, H.; Kumar, J.; Marx, K.; Tripathy, S. Sol-Gel Encapsulated Light-Transducing Protein Phycoerythrin - a New Biomaterial. Chem Mat 1995, 7 (10), 1779-1783.

(11) Lim, M. H.; Stein, A. Comparative Studies of Grafting and Direct Syntheses of Inorganic-Organic Hybrid Mesoporous Materials. Chem Mater 1999, 11 (11), 3285-3295.

(12) Moreau, J.J.E.; Vellutini, L.; Wong Chi Man, M.; Bied, C. New Hybrid Organic-Inorganic Solids with Helical Morphology via H-Bond Mediated Sol-Gel Hydrolysis of Silyl Derivatives of Chiral (R,R)- or (S,S)-Diureidocyclohexane. J. Am. Chem. Soc. 2001, 123, 1509-1510.

(13) Arrachart, G.; Carcel, C.; Trens, P.; Moreau, J.J.E.; Wong Chi Man, M. Silylated Melamine and Cyanuric Acid as Precursors for Imprinted and Hybrid Silica Materials with Molecular Recognition Properties. Chemistry a European Journal, 2009, 15(25), 6279-6288. 
(14) Arrachart, G.; Bendjerriou, A; Carcel, C.; Trens, P.; Moreau, J.J.E.; Wong Chi Man, M. Influence of the alkyl linker in the structuring of bridged silsesquioxanes obtained by selfrecognition properties New Journal of Chemistry 2010, 34(7), 1436-1440.

(15) Creff, G.; Arrachart, G.; Hermet, P.; Wadepohl, H.; Almairac, R.; Maurin, D.; Sauvajol, J.-L.; Carcel, C.; Moreau, J. J. E.; Dieudonne, P.; et al. Investigation on the Vibrational and Structural Properties of a Self-Structured Bridged Silsesquioxane. Phys Chem Chem Phys 2012, $14(16), 5672-5679$.

(16) Zhang, Q.; Ariga, K.; Okabe, A.; Aida, T. A Condensable Amphiphile with a Cleavable Tail as a "Lizard" Template for the Sol-Gel Synthesis of Functionalized Mesoporous Silica. J Am Chem Soc 2004, 126 (4), 988-989.

(17) Alauzun, J.; Besson, E.; Mehdi, A.; Reye, C.; Corriu, R. J. P. Reversible Covalent Chemistry of CO2: An Opportunity for Nano-Structured Hybrid Organic-Inorganic Materials. Chem. Mater. 2008, 20 (2), 503-513.

(18) Mouawia, R.; Mehdi, A.; Reyé, C.; Corriu, R. Direct Synthesis of Ordered and Highly Functionalized Organosilicas Containing Carboxylic Acid Groups. J. Mater. Chem. 2007, 17 (7), $616-618$.

(19) Mouawia, R.; Mehdi, A.; Reyé, C.; Corriu, R. J. P. From Simple Molecules to Highly Functionalised Lamellar Materials. J. Mater. Chem. 2008, 18 (17), 2028-2035.

(20) Besnard, R.; Cambedouzou, J.; Arrachart, G.; Diat, O.; Pellet-Rostaing, S. Self-Assembly of Condensable "Bola-Amphiphiles" in Water/Tetraethoxysilane Mixtures for the Elaboration of Mesostructured Hybrid Materials. Langmuir 2013, 29 (33), 10368-10375. 
(21) Besnard, R.; Arrachart, G.; Cambedouzou, J.; Pellet-Rostaing, S. Structural Study of Hybrid Silica Bilayers from "bola-Amphiphile" Organosilane Precursors: Catalytic and Thermal Effects. RSC Adv. 2015, 5 (71), 57521-57531.

(22) Jal, P. K.; Patel, S.; Mishra, B. K. Chemical Modification of Silica Surface by Immobilization of Functional Groups for Extractive Concentration of Metal Ions. Talanta 2004, $62(5), 1005-1028$.

(23) Yokoi, T.; Yoshitake, H.; Yamada, T.; Kubota, Y.; Tatsumi, T. Amino-Functionalized Mesoporous Silica Synthesized by an Anionic Surfactant Templating Route. J. Mater. Chem. 2006, $16(12), 1125-1135$.

(24) Charlot, A.; Mourabit, S. E.; Goettmann, F.; Arrachart, G.; Turgis, R.; Grandjean, A. From Phosphate Rocks to Uranium Raw Materials: Hybrid Materials Designed for Selective Separation of Uranium from Phosphoric Acid. RSC Adv. 2014, 4 (109), 64138-64141.

(25) Utting, K. A.; Macquarrie, D. J. Silica-Supported Imines as Mild, Efficient Base Catalysts. New J. Chem. 2000, 24 (8), 591-595.

(26) Clark, J. H.; Macquarrie, D. J. Catalysis of Liquid Phase Organic Reactions Using Chemically Modified Mesoporous Inorganic Solids. Chem. Commun. 1998, No. 8, 853-860.

(27) Li, Y.; Beck, R.; Huang, T.; Choi, M. C.; Divinagracia, M. Scatterless Hybrid Metalsingle-Crystal Slit for Small-Angle X-Ray Scattering and High-Resolution X-Ray Diffraction. J. Appl. Crystallogr. 2008, 41 (6), 1134-1139. 
(28) Pichon, B. P.; Wong Chi Man, M.; Bied, C.; Moreau, J. J. E. A Simple Access to $\omega-$ Aminoalkyltrialkoxysilanes: Tunable Linkers for Self-Organised Organosilicas. J Organomet Chem 2005, 1126-1130.

(29) Tanev, P. T.; Pinnavaia, T. J. A Neutral Templating Route to Mesoporous Molecular Sieves. Science 1995, 267 (5199), 865-867.

(30) Macquarrie, D. J. Direct Preparation of Organically Modified MCM-Type Materials. Preparation and Characterisation of Aminopropyl-MCM and 2-Cyanoethyl-MCM. Chem. Commun. 1996, No. 16, 1961-1962.

(31) Broome, F. K.; Hoerr, C. W.; Harwood, H. J. The Binary Systems of Water with Dodecylammonium Chloride and Its N-Methyl Derivatives. J. Am. Chem. Soc. 1951, 73 (7), $3350-3352$.

(32) Karlsson, S.; Friman, R.; Björkqvist, M.; Lindström, B.; Backlund, S. Phase Behavior and Characterization of the System Acetic Acid-Dodecylamine-Water. Langmuir 2001, 17 (12), $3573-3578$.

(33) Hartmann, P. C.; Dieudonné, P.; Sanderson, R. D. Self-Assembly and Influence of the Organic Counterion in the Ternary Systems Dodecylamine/acrylic Acid/water and Dodecylamine/methacrylic Acid/water. J. Colloid Interface Sci. 2005, 284 (1), 289-297.

(34) Yang, X.; Zhao, N.; Zhou, Q.; Wang, Z.; Duan, C.; Cai, C.; Zhang, X.; Xu, J. Facile Preparation of Hollow Amino-Functionalized Organosilica Microspheres by a Template-Free Method. J. Mater. Chem. 2012, 22 (34), 18010-18017. 
(35) Kind, L.; Plamper, F. A.; Göbel R.; Mantion, A,; Müller, A. H. E.; Pieles, U.; Andreas Taubert, A.; Meier, W., Langmuir, 2009, 25 (12), 7109-7115.

(36) Bougis, K.; Rubim, R. L.; Ziane, N.; Peyencet, J.; Bentaleb, A.; Février, A.; Oliveira, C. L. P.; Oliveira, E. A. de; Navailles, L.; Nallet, F. Stabilising Lamellar Stacks of Lipid Bilayers with Soft Confinement and Steric Effects. Eur. Phys. J. E 2015, 38 (7), 1-10.

(37) Katagiri, K.; Hashizume, M.; Ariga, K.; Terashima, T.; Kikuchi, J. Preparation and Characterization of a Novel Organic-Inorganic Nanohybrid "Cerasome" Formed with a Liposomal Membrane and Silicate Surface. Chem. - Eur. J. 2007, 13 (18), 5272-5281.

(38) Ibrahim M, N. A. Density Functional Theory and FTIR Spectroscopic Study of Carboxyl Group. Indian J Pure Appl Phys. Indian J. Pure Appl. Phys. 2005, 34 (12), 911-917.

(39) Riegel, B.; Kiefer, W.; Hofacker, S.; Schottner, G. FT-Raman-Spectroscopic Investigations of the System Glycidoxypropyltrimethoxysilane /Aminopropyltriethoxy-silane. J. Sol-Gel Sci. Technol. 1998, 13 (1-3), 385-390.

(40) Bantignies, J. L.; Vellutini L.; Maurin, D.; Hermet, P.; Dieudonné, P.; Wong Chi Man, M.; Bartlett, J.R.; Bied, C.; Sauvajol, J-L.; Moreau, J. J. Phys. Chem. B, 2006, 110 (32), 15797-15802.

(41) Orel, B.; Jese, R.; Vilcnik, A.; Stangar U. L. Hydrolysis and Solvolysis of Methyltriethoxysilane Catalyzed with $\mathrm{HCl}$ or Trifluoroacetic Acid: IR Spectroscopic and Surface Energy Studies. J. Sol-Gel Sci. Technol. 2005, 34, 251-265.

(42) Kaneko, Y.; Iyi, N.; Matsumoto, T.; Fujii, K.; Kurashima, K.; Fujita, T. Synthesis of IonExchangeable Layered Polysiloxane by Sol-gel Reaction of Aminoalkyltrialkoxysilane: A New Preparation Method for Layered Polysiloxane Materials. J. Mater. Chem. 2003, 13 (9), 2058-2060. 
(43) Kaneko, Y.; Iyi, N.; Kurashima, K.; Matsumoto, T.; Fujita, T.; Kitamura, K. HexagonalStructured Polysiloxane Material Prepared by Sol-Gel Reaction of Aminoalkyltrialkoxysilane without Using Surfactants. Chem. Mater. 2004, 16 (18), 3417-3423.

(44) Kaneko, Y.; Preparation of Ionic Silsesquioxanes with Regular Structures and Their Hybridization. Int. J. Polym. Sci. 2012, 2012 (1687-9422).

(45) Nagarajan, R. Molecular Packing Parameter and Surfactant Self-Assembly: The Neglected Role of the Surfactant Tail. Langmuir 2002, 18 (1), 31-38.

(46) Fedors, R. F. A Method for Estimating Both the Solubility Parameters and Molar Volumes of Liquids. Polym Eng Sci 1974, 14 (2), 147-154.

(47) Nagarajan, R. Self-Assembly of Bola Amphiphiles. Chem. Eng. Comm. 1987, 55, 251273. 


\section{Table of Contents/Abstract Graphics}

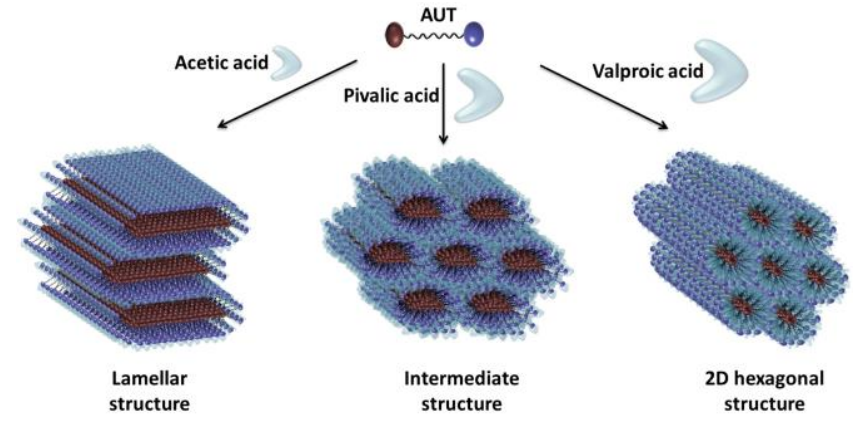

